\title{
A novel three-step approach for predicting the imminent onset of preeclampsia within 4 weeks after blood sampling at 19-31 weeks of gestation
}

\author{
Chikako Hirashima $^{1,2}$, Akihide Ohkuchi ${ }^{1,2}$, Kayo Takahashi ${ }^{1,2}$, Hirotada Suzuki ${ }^{1}$, Shigeki Matsubara ${ }^{1}$ \\ and Mitsuaki Suzuki ${ }^{1}$
}

Our aim was to develop a novel screening method to detect the imminent onset of preeclampsia (PE) within 4 weeks after blood sampling. We prospectively collected data regarding past history of PE/gestational hypertension (GH), blood pressure levels at 16-23 weeks and plasma levels of soluble fms-like tyrosine kinase 1 (sFIt-1) and placental growth factor (PIGF) twice at 19-31 weeks, which were measured using an automated electrochemiluminescence immunoassay. We found that a three-step approach by sequential selection using maternal factors, including a past history of PE/GH or blood pressure levels $\geqslant 120 / 80 \mathrm{~mm} \mathrm{Hg}$ at $16-23$ weeks (first step), followed by plasma levels of PIGF in the $<5$ th percentile (second step) and plasma levels of sFIt-1 in the $\geqslant 95$ th percentile (third step) yielded both high sensitivity and specificity. The imminent onset of PE occurred in 2 of $1199(0.2 \%)$ women recruited at 19-25 weeks and in 6 of $798(0.8 \%)$ women recruited at 26-31 weeks. The sensitivity, specificity, positive likelihood ratio ( $95 \%$ confidence interval), negative likelihood ratio (95\% confidence interval), positive predictive value and negative predictive value of the three-step approach for predicting the imminent onset of PE at 19-25 weeks were $100 \%, 99.8 \%, 599(150-2390), 0 \%, 50 \%$ and $100 \%$, respectively; and those at $26-31$ weeks were $83 \%, 99.1 \%, 94(42-214), 0.17(0.03-1.01), 42 \%$ and $99.9 \%$, respectively. In conclusion, the three-step approach is a highly sensitive and specific screening method for detecting the imminent onset of PE within 4 weeks after blood sampling at 19-31 weeks of gestation.

Hypertension Research (2014) 37, 519-525; doi:10.1038/hr.2014.31; published online 6 March 2014

Keywords: angiogenesis factor; blood pressure; prediction; preeclampsia

\section{INTRODUCTION}

Preeclampsia (PE), which develops in almost $3.5 \%$ of primiparous women and $2.0 \%$ of multiparous women in Japan, ${ }^{1}$ is a major complication of pregnancy associated with significant morbidity and mortality of both the fetus and mother., ${ }^{2,3}$ Several prospective studies have revealed that multivariate models using maternal factors and circulating biomarkers, including angiogenesis-related factors such as the soluble fms-like tyrosine kinase 1 (sFlt-1), placental growth factor (PlGF) and soluble endoglin, are promising for the prediction of PE. ${ }^{4,5}$ In some models, the positive likelihood ratio $(\mathrm{LR}+)$ for the prediction of early-onset PE exceeded $10,{ }^{6-9}$ which is an ideal level for clinically useful screening; ${ }^{10,11}$ however, this criterion using only the $\mathrm{LR}+$ does not consider the other important criteria of sensitivity (SE) and the positive predictive value (PPV). Therefore, in the current study, we thought that not only an LR $+\geqslant 10$ but also an $\mathrm{SE} \geqslant 0.80$ and a PPV $\geqslant 0.20$ should be required for the ideal level of clinically useful screening for PE.
To develop a new early diagnostic method with a high SE, LR + and PPV, we started with the following three hypotheses. First, the imminent onset of PE within 4 weeks after blood sampling might be a better primary endpoint than earlier onset of PE because the circulating levels of sFlt- 1 indicate a more rapid increase within 5 weeks before the onset of PE. ${ }^{12}$ Second, because blood pressure (BP) $\geqslant 120 / 80 \mathrm{~mm} \mathrm{Hg}$ at $16-23$ weeks is a risk factor for PE, having a relatively high SE of $76 \%,{ }^{13}$ the initial selection of high-risk women for PE should be performed using a BP $\geqslant 120 / 80 \mathrm{~mm} \mathrm{Hg}$ and/or a past history of PE/gestational hypertension $(\mathrm{GH})$, which is a wellknown risk factor for $\mathrm{PE} / \mathrm{GH}$; this probably contributes to ruling out pregnant women at very low risk for developing PE later during the gestational period. ${ }^{14}$ Third, sequential selection by both the plasma levels of PlGF followed by the plasma levels of sFlt-1 might achieve a high specificity (SP) while maintaining the SE because almost all preeclamptic women with onset at $<36$ weeks showed abnormalities in both the serum/plasma levels of PlGF and sFlt-1 after the onset of

${ }^{1}$ Department of Obstetrics and Gynecology, Jichi Medical University School of Medicine, Tochigi, Japan

${ }^{2}$ These authors contributed equally to this work.

Correspondence: Dr A Ohkuchi, Department of Obstetrics and Gynecology, Jichi Medical University School of Medicine, 3311-1 Yakushiji, Shimotsuke-shi, Tochigi 329-0498, Japan. 
$\mathrm{PE}^{4,15}$ and because both the levels of PlGF and sFlt- 1 in women with a later occurrence of PE significantly deviated from those in normal pregnant women at least 5 weeks before the onset. ${ }^{12}$

This is a post hoc analysis of a previous work. ${ }^{16}$ In the current study, we developed a novel three-step approach for predicting the imminent onset of PE within 4 weeks after blood sampling by sequential selection using maternal factors including a maternal BP $\geqslant 120 / 80 \mathrm{~mm} \mathrm{Hg}$ at $16-23$ weeks and/or a past history of $\mathrm{PE} / \mathrm{GH}$ (first step), followed by specific PlGF (second step) and sFlt-1 plasma levels (third step). Our aim was to evaluate the three-step approach for predicting the imminent onset of PE within 4 weeks after blood sampling at 19-31 weeks of gestation.

\section{METHODS}

\section{Subjects}

We obtained written informed consent from all the women enrolled in this study as well as approval from the Ethics Committee of our institute. The study subjects were almost all Japanese women with singleton pregnancies. They consisted of 1724 unselected pregnant women who were recruited into the prospective cohort study in our tertiary center between April 2004 and September 2008. A total of 123 women were excluded because their PE outcomes or infant data could not be obtained. In the remaining 1601 women, 1199 blood samples were collected at 19-25 weeks of gestation, and 798 blood samples were collected at 26-31 weeks of gestation in a total of 1239 women. PE occurred in $35(2.8 \%)$ (onset gestational weeks: median (interquartile range), 35.4 (31.5-37.5)), early-onset PE with onset at $<32$ weeks of gestation in $11(0.9 \%)$ (onset gestational weeks: median (interquartile range), 29.8 (26.5-31.5)), GH in 20 (1.6\%) and a small-for-gestational-age (SGA) infant in $141(11.4 \%)$ of the 1239 women. All the subjects had been previously used to evaluate the onset threshold of the sFlt-1/PlGF ratio for predicting the imminent onset of PE and to evaluate whether the plasma levels of PlGF and sFlt-1 were valuable for predicting PE in general, PE with onset at $<36$ weeks, $<34$ weeks and $<32$ weeks, and GH and an SGA infant. ${ }^{16}$

\section{Blood pressure in pregnancy and the puerperal period}

Systolic blood pressure and diastolic blood pressure were measured with an Omron HEM-906 automated digital oscillometric sphygmomanometer (OMRON Healthcare, Kyoto, Japan), according to standard procedures. The mean values of two systolic blood pressure and diastolic blood pressure readings at 16-19 and 20-23 weeks of gestation were documented for each participant.

\section{Measurement of indices in uterine artery Doppler}

The details of the measurement of the indices in uterine artery Doppler have been provided previously. ${ }^{16-18}$ The normal reference values at $16-23$ weeks were determined in our previous study, ${ }^{17}$ and the cutoff values of the mean notch depth index (mNDI) and mean pulsatility index (mPI) for predicting PE were set at the 90th percentile for the distributions of the mNDI and mPI at 16-23 weeks in normal pregnant women. ${ }^{17}$

\section{Measurement of sFlt-1 and PlGF plasma levels}

The details of the measurements of the sFlt- 1 and PlGF plasma levels have been provided previously. ${ }^{16}$ Briefly, blood samples were collected into tubes containing ethylenediaminetetraacetic acid, centrifuged at $4{ }^{\circ} \mathrm{C}$ at 2500 r.p.m. for $15 \mathrm{~min}$ and stored at $-20{ }^{\circ} \mathrm{C}$ until use. The ECLIAs of sFlt- 1 and PlGF using the Elecsys sFlt-1 and PlGF (Roche Diagnostics, Penzberg, Germany) assays were performed using a Modular Analytics E 170 (Roche Diagnostics) according to the manufacturer's instructions.

Definition of cutoff points of PIGF, sFlt-1 and the sFlt-1/PIGF ratio Details of the definition of the cutoff points of the plasma levels of sFlt-1 and PlGF have been provided previously. ${ }^{16}$ The cutoff levels of sFlt-1 and PlGF at 19-31 weeks were set at the 95th and 5th percentile, respectively. In brief, an abnormal sFlt-1 at 19-25 weeks was defined as $\geqslant 2540 \mathrm{pg} \mathrm{ml}^{-1}$ and that at 26-31 weeks was defined as $\geqslant 2560 \mathrm{pg} \mathrm{ml}^{-1}$; an abnormal PlGF at 19-25 weeks was defined as $<128 \mathrm{pg} \mathrm{ml}^{-1}$ and that at $26-31$ weeks was defined as $<177 \mathrm{pg} \mathrm{ml}^{-1}$.

\section{Definitions of PE, GH and an SGA infant}

We defined PE and GH according to the definition and classification of PIH (2004) of the Japan Society for the Study of Hypertension in Pregnancy (JSSHP). ${ }^{19}$ In brief, PE was defined as hypertension with proteinuria occurring after 20 weeks of gestation. Superimposed PE was defined as chronic hypertension diagnosed prior to pregnancy or prior to 20 weeks of gestation, with proteinuria emerging afterward. Superimposed PE was included in the PE category in this study. GH was defined as hypertension without proteinuria occurring after 20 weeks of gestation. Hypertension was defined as an systolic blood pressure that was $\geqslant 140 \mathrm{~mm} \mathrm{Hg}$ and/or a diastolic blood pressure that was $\geqslant 90 \mathrm{~mm} \mathrm{Hg}$ on 2 occasions $\geqslant 4 \mathrm{~h}$ apart. Proteinuria was defined as $300 \mathrm{mg}$ per day from a 24 -hour urine collection. If only a test tape was available, repeated semi-quantitative test results of $1+$, which represented $30 \mathrm{mg} \mathrm{dl}^{-1}$ protein or more, were considered to constitute a positive result. PE with an onset earlier than 32 weeks of gestation was defined as early-onset PE. An SGA infant was defined as having a birth weight below the 10th percentile for the Japanese population. ${ }^{20}$

\section{Two-step and three-step approaches in different settings}

In the current study, we analyzed both a novel two-step approach and a three-step approach for predicting the imminent onset of PE within 4 weeks after blood sampling. We evaluated two types of two-step approach (i and ii) and six types of three-step approaches (iii-viii) as follows.

First, we evaluated the early diagnostic performance for predicting the imminent onset of PE within 4 weeks after blood sampling at 19-25 weeks and 26-31 weeks using the two types of the two-step approach: (i) the first step was determining the P1GF plasma level, followed by the second step, determining the sFlt-1 plasma level, and (ii) first step was determining the sFlt-1 plasma level, followed by the second step, determining the P1GF plasma level.

Second, we evaluated the early diagnostic performance for predicting the imminent onset of PE within 4 weeks after blood sampling at 19-25 weeks and 26-31 weeks using two variations of the three-step approach: (iii) the first step was to determine the maternal factors, including BP levels of $\geqslant 120 / 80 \mathrm{~mm} \mathrm{Hg}$ at 16-23 weeks or a past history of $\mathrm{PE} / \mathrm{GH}$, followed by the second step, determining the plasma level of PIGF, and the third step, determining the plasma level of sFlt-1, and (iv) the first step was to determine the maternal factors, followed by the second step, determining the plasma level of sFlt-1, and the third step, determining the plasma level of PlGF.

Third, we evaluated the early diagnostic performance of our three-step approach for predicting the imminent onset of PE within 4 weeks after blood sampling at 19-25 weeks and 26-31 weeks. When the first step of the threestep approach was the maternal factors, including BP levels of $\geqslant 120 / 80 \mathrm{~mm}$ $\mathrm{Hg}$ at $16-23$ weeks, a past history of $\mathrm{PE} / \mathrm{GH}$, or $\mathrm{mPI} \geqslant 90$ th percentile and/or mNDI $\geqslant 90$ th percentile, the two types of three-step approach were as follows: (v) the first step was to determine the maternal factors, including BP levels of $\geqslant 120 / 80 \mathrm{~mm} \mathrm{Hg}$ at $16-23$ weeks, a past history of $\mathrm{PE} / \mathrm{GH}$, or $\mathrm{mPI} \geqslant 90$ th percentile and/or mNDI $\geqslant 90$ th percentile, followed by the second step, determining the PlGF plasma level, and the third step, determining the sFlt-1 plasma level, and (vi) the first step was to determining the maternal factors, followed by the second step, determining the plasma level of sFlt-1, and the third step, determining the plasma level of PlGF. The details of the results and a discussion are included in the Supplementary Information.

Finally, we evaluated the early diagnostic performance of our three-step approach for predicting the imminent onset of PE within 4 weeks after blood sampling across 19-31 weeks in 758 women in whom both the sFlt- 1 and PlGF plasma levels were measured twice at 19-25 and 26-31 weeks and in 19 women with an early delivery of $<32$ weeks in whom both the sFlt- 1 and PlGF plasma levels were measured only once at 19-25 weeks. When the first step of the three-step approach was the maternal factors, including BP levels of $\geqslant 120 / 80 \mathrm{~mm} \mathrm{Hg}$ at $16-23$ weeks or a past history of PE/GH, the two types of the three-step approach were as follows: (vii) the first step was to determine the maternal factors, including BP levels of $\geqslant 120 / 80 \mathrm{~mm} \mathrm{Hg}$ at $16-23$ weeks or a past history of $\mathrm{PE} / \mathrm{GH}$, followed by the second step, determining the plasma 
level of PlGF, and the third step, determining the plasma level of sFlt-1, and (viii) the first step was to determine the maternal factors, followed by the second step, determining the plasma level of sFlt-1, and the third step, determining the plasma level of PlGF. The details of the results and a discussion are included in the Supplementary Information.

In addition, we constructed survival curves for (a) the women not selected by the first step, (b) the women not selected by the second step, (c) the women not selected by the third step, and (d) the women selected by all steps when the first step of the three-step approach was the maternal factors, including BP levels of $\geqslant 120 / 80 \mathrm{~mm} \mathrm{Hg}$ at $16-23$ weeks or a past history of PE/GH in the three-step approach (type iii). The details of the results and a discussion are included in the Supplementary Information.

\section{Statistics}

The results are presented as the mean \pm s.d. For the plasma sFlt-1, PlGF and the sFlt-1/PlGF ratio, the statistical testing was conducted after a logarithmic transformation. An unpaired $t$-test was used to compare two continuous variables; the $\chi^{2}$ test or Fisher's exact test was used to compare the categorical data; and these analyses were performed using the software included in the IBM SPSS software package (version 21, IBM, Armonk, NY, USA). The LR + and the $95 \%$ confidence interval were calculated using the Confidence Interval Calculator (created by Rob Herbert at the University of Sydney). ${ }^{21}$ An LR + $\geqslant 10,{ }^{10,11}$ an SE $\geqslant 0.8$ and a PPV $\geqslant 0.20$ were considered clinically useful for an early diagnostic test. $P<0.05$ was considered significant.

\section{RESULTS}

\section{Basic characteristics}

In the women with $\mathrm{PE}$, the frequencies of a past history of $\mathrm{PE} / \mathrm{GH}$, a family history of hypertension, obesity, chronic hypertension, systolic blood pressure $\geqslant 120 \mathrm{~mm} \mathrm{Hg}$, diastolic blood pressure $\geqslant 80 \mathrm{~mm} \mathrm{Hg}$, $\mathrm{mPI} \geqslant 90$ th percentile and an mNDI $\geqslant 90$ th percentile were significantly greater than in normal pregnant women (Table 1). In the women with $\mathrm{PE}$, preterm delivery occurred more frequently than in normal pregnant women, and an SGA infant was more frequently born than in normal pregnant women.

Table 1 Maternal and neonatal characteristics in normal pregnant women and preeclamptic women

\begin{tabular}{|c|c|c|c|c|c|}
\hline Characteristics & Normal pregnant women ${ }^{\mathrm{a}}(\mathrm{n}=1184)$ & Women with $P E(\mathrm{n}=35)$ & $L R+(95 \% C l)$ & Missing values (normal/PE) & $\mathrm{P}$-value \\
\hline \multicolumn{6}{|l|}{ Maternal } \\
\hline Age (year) & $32.7 \pm 5.0$ & $34.3 \pm 5.6$ & - & 0 & 0.064 \\
\hline Age $\geqslant 40$ year $(\%)$ & $79(6.7)$ & $4(11.4)$ & $1.7(0.67-4.4)$ & 0 & 0.293 \\
\hline Race: Japanese (\%) & $11(0.9)$ & $0(0)$ & NC & 0 & 1.000 \\
\hline Nulliparous women (\%) & $563(47.6)$ & $18(51.4)$ & $1.1(0.78-1.5)$ & 0 & 0.732 \\
\hline Past history of PE/GH (\%) & $46(3.9)$ & $7(20.0)$ & $5.1(2.5-11)$ & $1 / 0$ & $<0.001$ \\
\hline Family history of hypertension (\%) & $277(23.7)$ & $17(48.6)$ & $2.0(1.4-2.9)$ & $17 / 0$ & 0.002 \\
\hline Current smoker (\%) & $57(5.1)$ & $4(11.4)$ & $2.4(0.86-5.8)$ & $75 / 0$ & 0.111 \\
\hline Pre-pregnancy BMI (mean \pm s.d., $\mathrm{kg} \mathrm{m}^{-2}$ ) & $22.3 \pm 4.2$ & $25.2 \pm 5.9$ & - & $4 / 0$ & 0.006 \\
\hline Obesity (\%) & $222(18.8)$ & $17(48.6)$ & $2.6(1.8-3.7)$ & $4 / 0$ & $<0.001$ \\
\hline Chronic hypertension (\%) & $17(1.4)$ & $8(22.9)$ & $16(7.4-34)$ & 0 & $<0.001$ \\
\hline SBP at $16-23$ weeks $(\mathrm{mm} \mathrm{Hg})$ & $116.0 \pm 13.6$ & $134.8 \pm 18.9$ & - & $6 / 0$ & $<0.001$ \\
\hline DBP at $16-23$ weeks $(\mathrm{mm} \mathrm{Hg})$ & $67.3 \pm 9.4$ & $81.0 \pm 11.5$ & - & $6 / 0$ & $<0.001$ \\
\hline MAP at $16-23$ weeks $(\mathrm{mm} \mathrm{Hg})$ & $83.6 \pm 10.3$ & $98.9 \pm 13.7$ & - & $6 / 0$ & $<0.001$ \\
\hline SBP at $16-23$ weeks $\geqslant 120 \mathrm{~mm} \mathrm{Hg}(\%)$ & 409 (34.7) & $28(80.0)$ & $2.3(1.9-2.8)$ & $5 / 0$ & $<0.001$ \\
\hline DBP at $16-23$ weeks $\geqslant 80 \mathrm{~mm} \mathrm{Hg}(\%)$ & $110(9.3)$ & $17(48.6)$ & $5.2(3.5-7.6)$ & $5 / 0$ & $<0.001$ \\
\hline MAP at $16-23$ weeks $\geqslant 90 \mathrm{~mm} \mathrm{Hg}(\%)$ & $285(24.2)$ & $25(71.4)$ & $3.0(2.3-3.7)$ & $5 / 0$ & $<0.001$ \\
\hline \multicolumn{6}{|l|}{ Blood pressure levels at 16-23 weeks ${ }^{b}$} \\
\hline Optimal (\%) & $769(65.2)$ & $7(20.0)$ & - & $5 / 0$ & $<0.001$ \\
\hline Normal (\%) & $236(20.0)$ & $7(20.0)$ & & & \\
\hline High-normal (\%) & $115(9.8)$ & $7(20.0)$ & & & \\
\hline Hypertension (\%) & $59(5.0)$ & $14(40.0)$ & & & \\
\hline $\mathrm{mPI}$ at $16-23$ weeks $\geqslant 90$ th percentile $(\%)^{c}$ & $149(13.1)$ & $16(45.7)$ & $3.5(2.4-5.2)$ & $45 / 0$ & $<0.001$ \\
\hline $\mathrm{mNDI}$ at $16-23$ weeks $\geqslant 90$ th percentile $(\%)^{\mathrm{c}}$ & $123(10.8)$ & $15(42.9)$ & $4.0(2.6-6.0)$ & $45 / 0$ & $<0.001$ \\
\hline \multicolumn{6}{|l|}{ Combination of $\mathrm{mPI}$ and $\mathrm{mNDI}$} \\
\hline $\mathrm{mPI}<90$ th and mNDI <90th (\%) & $940(82.5)$ & $17(48.6)$ & - & $45 / 0$ & $<0.001$ \\
\hline only $\mathrm{mPI} \geqslant 90$ th or only $\mathrm{mNDI} \geqslant 90$ th (\%) & $126(11.1)$ & $5(14.3)$ & & & \\
\hline both $\mathrm{mPI} \geqslant 90$ and $\mathrm{mNDI} \geqslant 90$ th (\%) & $73(6.4)$ & $13(37.1)$ & & & \\
\hline \multicolumn{6}{|l|}{ Neonatal } \\
\hline Gestational age at delivery (week) & $38.8 \pm 2.1$ & $36.1 \pm 4.3$ & - & 0 & $<0.001$ \\
\hline Preterm delivery (\%) & $117(9.9)$ & $16(45.7)$ & - & 0 & $<0.001$ \\
\hline Birth weight (g) & $2942 \pm 481$ & $2335 \pm 887$ & - & 0 & $<0.001$ \\
\hline Small-for-gestational-age infant $(\%)^{d}$ & $125(10.6)$ & $11(31.4)$ & - & 0 & $<0.001$ \\
\hline
\end{tabular}

Abbreviations: $\mathrm{BMI}$, body mass index; $\mathrm{Cl}$, confidence interval; DBP, diastolic blood pressure; GH, gestational hypertension; LR +, positive likelihood ratio; MAP, mean arterial pressure; mNDI, mean notch depth index; mPI, mean pulsatility index; PE, preeclampsia; SBP, systolic blood pressure; wk, weeks of gestation.

aNormal pregnant women were defined as women not complicated with PE or GH.

'The blood pressure levels were categorized into four groups according to the Japanese Society of Hypertension (JSH) 2009: optimal, SBP $<120 \mathrm{~mm} \mathrm{Hg}$ and DBP $<80 \mathrm{~mm} \mathrm{Hg}$; normal, $\mathrm{SBP}<130 \mathrm{~mm} \mathrm{Hg}$ and $\mathrm{DBP}<85 \mathrm{~mm} \mathrm{Hg}$, but not optimal; high-normal, SBP $<140 \mathrm{~mm} \mathrm{Hg}$ and $\mathrm{DBP}<90 \mathrm{~mm} \mathrm{Hg}$, but not either optimal or normal; hypertension, $\mathrm{SBP} \geqslant 140 \mathrm{~mm} \mathrm{Hg}$ or $\mathrm{DBP} \geqslant 90 \mathrm{~mm} \mathrm{Hg}$.

${ }^{c}$ The cutoff values of 90th percentiles of $\mathrm{mPI}$ and $\mathrm{mNDI}$ were selected according to the respective normal reference value in our previous paper.

${ }^{\mathrm{d} A}$ s small-for-gestational-age infant was defined as an infant having a birth weight below the 10th percentile for the Japanese population. 
In this cohort, $\mathrm{PE}$ occurred in $35(2.8 \%)$ women, early-onset $\mathrm{PE}$ with onset at $<32$ weeks of gestation occurred in $11(0.9 \%)$ women and late-onset $\mathrm{PE}$ with onset at $\geqslant 32$ weeks of gestation occurred in 24 (1.9\%) women; the median weeks (interquartile range) from the diagnosis to delivery in all $\mathrm{PE}$, early-onset $\mathrm{PE}$ and late-onset $\mathrm{PE}$ were 1.4 (0.7-3.1), $2.6(1.3-4.1)$ and $1.1(0.4-2.0)$ weeks.

Two-step approach for predicting the imminent onset of PE within 4 weeks after blood sampling

At 19-25 weeks, the two types of two-step approaches (type i and ii) fulfilled the three criteria of a clinically useful early diagnostic test: a high SE of 1.00, a high LR + of 599 and a high PPV of 0.50 (Table 2). At 26-31 weeks, the two types of two-step approaches also fulfilled the three criteria of a clinically useful early diagnostic test: a high SE of 0.83 , a high LR + of 83 and a high PPV of 0.39 (Table 3).

Three-step approach for predicting the imminent onset of PE within 4 weeks after blood sampling when the first step of the three-step approach was determining the maternal factors, including BP levels of $\geqslant 120 / 80 \mathrm{~mm} \mathrm{Hg}$ at $16-23$ weeks or a past history of $\mathrm{PE} / \mathrm{GH}$

For the two types of the three-step approach (type iii and iv) at 19-25 weeks, both methods fulfilled the three criteria of a clinically useful early diagnostic test: a high SE of 1.00, a high LR + of 599 and a high PPV of 0.50 (Table 2). At 26-31 weeks, the two types of a three-step approach also fulfilled the three criteria of a clinically useful early diagnostic test: a high SE of 0.83 , a high LR + of 94 and a high PPV of 0.42 (Table 3 ).

As for the third three-step approach (type iii), Figures 1a-d show that only one woman without PE (one GH with onset at 7 weeks after sampling) and only one woman with PE with an onset at 8 weeks after sampling had both sFlt-1levels in the $\geqslant 95$ th percentile and PlGF levels in the $<5$ th percentile at 19-25 weeks and that only 7 women without PE (six normal pregnant women, and one woman with GH with onset at 4 weeks after sampling) had both sFlt-1 levels in the $\geqslant 95$ th percentile and PlGF levels in the $<5$ th percentile at 26-31 weeks. Figures $1 \mathrm{c}$ and d clearly show that both the sFlt- 1 and PlGF levels at 19-31 weeks in $88 \%$ of the cases (7/8) with the imminent onset of PE deviated from the normal reference range of each metric. In the three-step approach at 19-25 weeks and 26-31 weeks using the maternal factors as the first step, followed by the plasma level of PlGF as the second step and the plasma level of sFlt-1 as the third step, the SPs were markedly improved from the first step to the third step, retaining a high SE. The LR + also increased rapidly from the first step to the third step, yielding a very high LR + of $>90$ (Figure 2a). In addition, the subject numbers in the second

Table 2 Early diagnostic performances for predicting imminent onset of PE within 4 weeks after blood sampling, by two-step approach using plasma levels of PIGF and sFIt-1, and three-step approach using maternal factors, plasma levels of PIGF and sFIt-1, in 1199 pregnant women at 19-25 weeks, when the first step of the three-step approach was maternal factors, including BP levels of $\geqslant 120 / 80 \mathrm{~mm} \mathrm{Hg}$ at $16-23$ weeks or a past history of $\mathrm{PE} / \mathrm{GH}$

\begin{tabular}{|c|c|c|c|c|c|c|c|c|c|c|c|}
\hline Risk factors & Abnormal value & $T P$ & $F N$ & $F P$ & $T N$ & $S E$ & $S P$ & $L R+(95 \% C l)$ & $L R-(95 \% C l)$ & $P P V$ & NPV \\
\hline \multicolumn{12}{|l|}{ Two-step approach } \\
\hline \multicolumn{12}{|l|}{$P I G F \rightarrow s F / t-1$} \\
\hline First step ${ }^{a}$ & PIGF $<5$ th & 2 & 0 & 67 & 1130 & 1.00 & 0.94 & $18(14-23)$ & 0 & 0.029 & 1.000 \\
\hline Second step ${ }^{b}$ & sFlt-1 $\geqslant 95$ th & 2 & 0 & 2 & 1195 & 1.00 & 0.998 & $599(150-2390)$ & 0 & 0.500 & 1.000 \\
\hline \multicolumn{12}{|l|}{$s F I t-1 \rightarrow P I G F$} \\
\hline First step ${ }^{c}$ & sFlt-1 $\geqslant 95$ th & 2 & 0 & 66 & 1131 & 1.00 & 0.94 & $18(14-23)$ & 0 & 0.029 & 1.000 \\
\hline Second step ${ }^{d}$ & PIGF <5th & 2 & 0 & 2 & 1195 & 1.00 & 0.998 & $599(150-2390)$ & 0 & 0.500 & 1.000 \\
\hline \multicolumn{12}{|c|}{ Three-step approach } \\
\hline \multicolumn{12}{|c|}{ Maternal factors $\rightarrow$ PIGF $\rightarrow$ sFlt- 1} \\
\hline First stepe & $\mathrm{PH}$ of $\mathrm{PE} / \mathrm{GH}$, or $\mathrm{BP} \geqslant 120 / 80$ & 2 & 0 & 461 & 736 & 1.00 & 0.62 & $2.6(2.4-2.8)$ & 0 & 0.004 & 1.000 \\
\hline Second step ${ }^{f}$ & PIGF $<5$ th & 2 & 0 & 42 & 1155 & 1.00 & 0.97 & $29(21-38)$ & 0 & 0.045 & 1.000 \\
\hline Third stepg & sFlt-1 $\geqslant 95$ th & 2 & 0 & 2 & 1195 & 1.00 & 0.998 & $599(150-2390)$ & 0 & 0.500 & 1.000 \\
\hline \multicolumn{12}{|c|}{ Maternal factors $\rightarrow$ sFlt- $1 \rightarrow$ PIGF } \\
\hline First stepe & $\mathrm{PH}$ of $\mathrm{PE} / \mathrm{GH}$, or $\mathrm{BP} \geqslant 120 / 80$ & 2 & 0 & 461 & 736 & 1.00 & 0.62 & $2.6(2.4-2.8)$ & 0 & 0.004 & 1.000 \\
\hline Second step ${ }^{h}$ & sFlt-1 $\geqslant 95$ th & 2 & 0 & 23 & 1174 & 1.00 & 0.98 & $52(35-78)$ & 0 & 0.080 & 1.000 \\
\hline Third step ${ }^{i}$ & PIGF $<5$ th & 2 & 0 & 2 & 1195 & 1.00 & 0.998 & $599(150-2390)$ & 0 & 0.500 & 1.000 \\
\hline \multicolumn{12}{|c|}{ 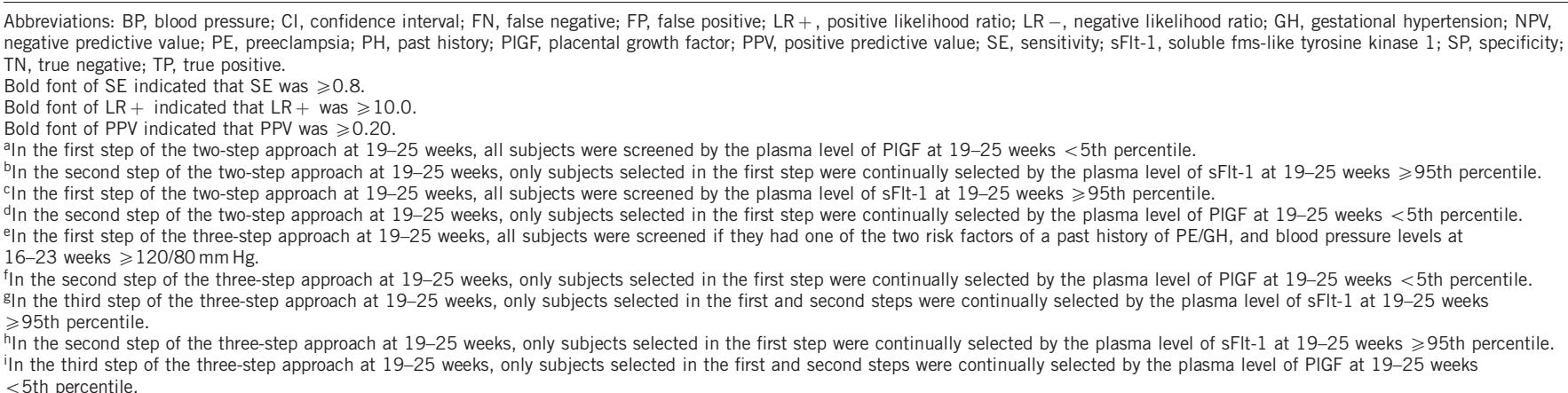 } \\
\hline
\end{tabular}


Table 3 Early diagnostic performances for predicting imminent onset of PE within 4 weeks after blood sampling, by two-step approach using plasma levels of PIGF and sFIt-1, and three-step approach using maternal factors, plasma levels of PIGF and sFIt-1, in 798 pregnant women at 26-31 weeks, when the first step of the three-step approach was maternal factors, including BP levels of $\geqslant 120 / 80 \mathrm{~mm} \mathrm{Hg}$ at $16-23$ weeks or a past history of $\mathrm{PE} / \mathrm{GH}$

\begin{tabular}{|c|c|c|c|c|c|c|c|c|c|c|c|}
\hline Risk factors & Abnormal value & $T P$ & $F N$ & $F P$ & $T N$ & $S E$ & $S P$ & $L R+(95 \% C l)$ & $L R-(95 \% C l)$ & $P P V$ & NPV \\
\hline \multicolumn{12}{|l|}{ Two-step approach } \\
\hline \multicolumn{12}{|l|}{$P I G F \rightarrow s F / t-1$} \\
\hline Second step ${ }^{b}$ & sFlt-1 $\geqslant 95$ th & 5 & 1 & 8 & 784 & 0.83 & 0.990 & $83(38-179)$ & $0.17(0.03-1.01)$ & 0.385 & 0.999 \\
\hline \multicolumn{12}{|l|}{ sFIt-1 $\rightarrow$ PIGF } \\
\hline \multicolumn{12}{|c|}{ Three-step approach } \\
\hline \multicolumn{12}{|c|}{ Maternal factors $\rightarrow$ PIGF $\rightarrow$ sFlt- 1} \\
\hline First stepe & $\mathrm{PH}$ of $\mathrm{PE} / \mathrm{GH}$, or $\mathrm{BP} \geqslant 120 / 80$ & 6 & 0 & 304 & 488 & 1.00 & 0.62 & $2.6(2.4-2.8)$ & 0 & 0.019 & 1.000 \\
\hline Second step $^{f}$ & PIGF $<5$ th & 5 & 1 & 24 & 768 & 0.83 & 0.97 & $28(16-47)$ & $0.17(0.03-1.03)$ & 0.172 & 0.999 \\
\hline Third stepg & sFIt- $1 \geqslant 95$ th & 5 & 1 & 7 & 785 & 0.83 & 0.991 & $94(42-214)$ & $0.17(0.03-1.01)$ & 0.417 & 0.999 \\
\hline \multicolumn{12}{|c|}{ Maternal factors $\rightarrow$ sFlt- $1 \rightarrow$ PIGF } \\
\hline Third step ${ }^{i}$ & PIGF $<5$ th & 5 & 1 & 7 & 785 & 0.83 & 0.991 & $94(42-214)$ & $0.17(0.03-1.01)$ & 0.417 & 0.999 \\
\hline
\end{tabular}

Abbreviations: BP, blood pressure; Cl, confidence interval; FN, false negative; FP, false positive; LR+, positive likelihood ratio; LR -, negative likelihood ratio; GH, gestational hypertension; NPV, negative predictive value; PE, preeclampsia; PH, past history; PIGF, placental growth factor; PPV, positive predictive value; SE, sensitivity; sFIt-1, soluble fms-like tyrosine kinase 1; SP, specificity; TN, true negative; TP, true positive.

Bold font of SE indicated that SE was $\geqslant 0.8$.

Bold font of $L R+$ indicated that $L R+$ was $\geqslant 10.0$

Bold font of PPV indicated that PPV was $\geqslant 0.20$

aIn the first step of the two-step approach at 26-31 weeks, all subjects were screened by the plasma level of PIGF at 26-31 weeks $<5$ th percentile.

bIn the second step of the two-step approach at $26-31$ weeks, only subjects selected in the first step were continually selected by the plasma level of sFlt- 1 at $26-31$ weeks $\geqslant 95$ th percentile.

In the first step of the two-step approach at 26-31 weeks, all subjects were screened by the plasma level of sFlt-1 at 26-31 weeks $\geqslant 95$ th percentile.

dIn the second step of the two-step approach at 26-31 weeks, only subjects selected in the first step were continually selected by the plasma level of PIGF at 26-31 weeks $<5$ th percentile.

eIn the first step of the three-step approach at 26-31 weeks, all subjects were screened if they had one of the three risk factors of a past history of PE/GH, and blood pressure levels at $16-23$ weeks $\geqslant 120 / 80 \mathrm{~mm} \mathrm{Hg}$.

fIn the second step of the three-step approach at 26-31 wk, only subjects selected in the first step were continually selected by the plasma level of PIGF at 26-31 wk $<5$ th percentile. gIn the third step of the three-step approach at 26-31 weeks, only subjects selected in the first and second steps were continually selected by the plasma level of sFIt-1 at 26-31 weeks $\geqslant 95$ th percentile.

hIn the second step of the three-step approach at 26-31 weeks, only subjects selected in the first step were continually selected by the plasma level of sFlt- 1 at $26-31$ weeks $\geqslant 95$ th percentile.

i In the third step of the three-step approach at 26-31 weeks, only subjects selected in the first and second steps were continually selected by the plasma level of PIGF at $26-31$ weeks

$<5$ th percentile.

step decreased to nearly $40 \%$, and the subject numbers in the third step decreased to nearly $4 \%$, yielding a cost reduction of $78 \%$ for performing the sFlt-1 and PlGF measurements in this three-step approach compared with an approach for measuring both sFlt-1 and PlGF in all subjects (Figure 2b).

\section{DISCUSSION}

In this study, we found that both the two-step approach and the three-step approach for predicting the imminent onset of PE within 4 weeks after blood sampling at 19-31 weeks showed a high SE, a high LR + and a high PPV.

The three-step approach for predicting the imminent onset of $\mathrm{PE}$ at 19-31 weeks showed a high SE, a high LR + and a high PPV. To the best of our knowledge, this is a novel method for predicting PE. In previous reports, we found that the onset threshold of the sFlt-1/PlGF ratio at 19-31 weeks for the onset of $\mathrm{PE}$ was able to predict the rapid onset of PE after blood sampling. ${ }^{4,16}$ In addition, the circulating levels of sFlt-1 revealed a more rapid increase within five weeks before the onset of PE. ${ }^{12}$ These findings indicated that the prediction of PE using the sFlt-1/PlGF ratio or the sFlt-1 level might be valid within $\sim 4-5$ weeks after blood sampling. Using the maternal characteristics as the first selection criteria resulted in a subject reduction of nearly $40 \%$, maintaining the SEs at 1.00 at both 19-25 weeks and 26-31 weeks, indicating that the first screening using BP levels of $\geqslant 120 / 80 \mathrm{~mm} \mathrm{Hg}$ or a past history of PE/GH may be useful for ruling out a low-risk pregnancy. The second selection by PlGF resulted in an additional subject reduction of nearly $3.6 \%$, maintaining the SEs at 1.00 at $19-25$ weeks and 0.83 at 26-31 weeks and markedly improving the SPs to 0.97 at both 19-25 weeks and 26-31 weeks. The third selection by the sFlt-1 levels resulted in excellent SE and SP results: the SE and SP at 19-25 weeks were 1.00 and 0.998, respectively, yielding an LR + of 599 and a PPV of 0.500 ; and the SE and SP at 26-31 weeks were 0.83 and 0.991 , respectively, yielding an LR + of 94 and a PPV of 0.417 . To the best of our knowledge, the early diagnostic performance for predicting the imminent onset of PE using either our two- or three-step approach was the best of the various combination models when the maternal characteristics and angiogenesis-related factors from the first to second trimesters were used to predict PE. ${ }^{5}$ We attempted another three-step approach using the three maternal characteristics of a $\mathrm{BP} \geqslant 120 / 80 \mathrm{~mm} \mathrm{Hg}$, a past history of $\mathrm{PE} / \mathrm{GH}$ or $\mathrm{mPI} \geqslant 90$ th percentile and/or an $\mathrm{mNDI} \geqslant 90$ th percentile as a first step; however, the SE, LR + and PPV were not improved (Supplementary Tables S1 and S2).

When we compared the cost performances for predicting the imminent onset of PE using the two approaches, the total cost for the 
Very small open circle (normal pregnancy, 。)

Small open square $(\mathrm{GH}, \square)$

Symbols accentuated by red bold line $(\circ, \square)$ : MF $(+)$, PIGF $<5^{\text {th }}$, and sFlt- $1 \geq 95^{\text {th }}$
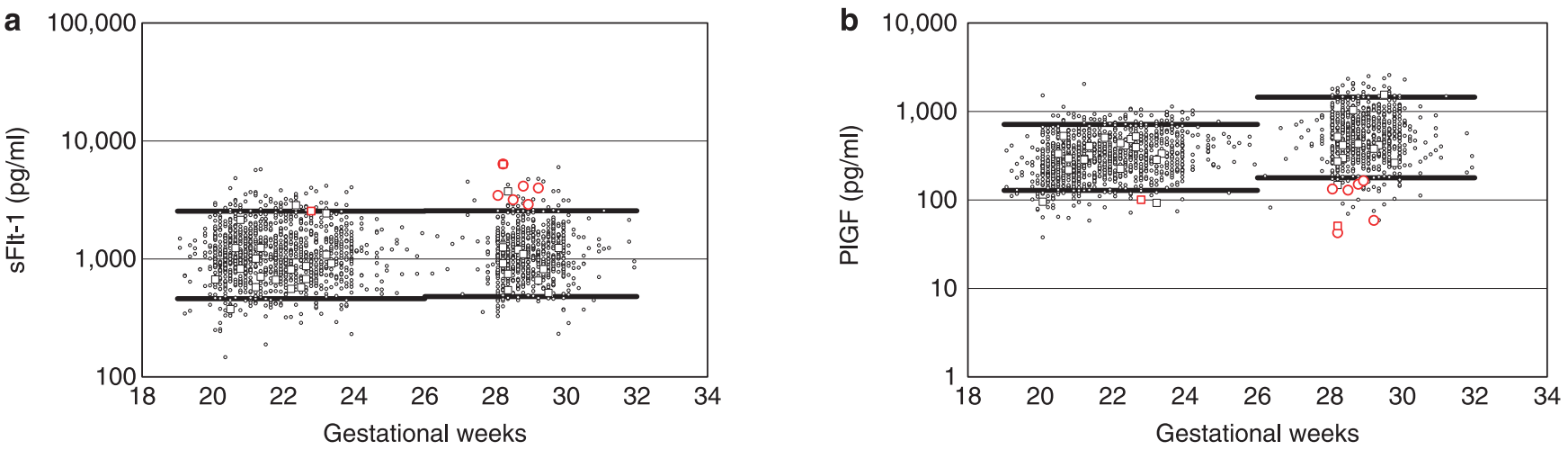

Small open triangle $(\Delta)$ : PE with onset at $\geq 36$ wks, but without imminent onset of PE

Intermediate-sized open triangle $(\triangle)$ : PE with onset at 32-35 wks, but without imminent onset of PE

Large open triangle $(\triangle)$ : PE with at $<32$ wks, but without imminent onset of $P E$

Yellow closed triangle $(, \Delta, \triangle)$ : imminent onset of $\mathrm{PE}$

Symbols accentuated by red bold line $(\triangle, \triangle)$ : MF $(+)$, PIGF $<5^{\text {th }}$, and sFlt-1 $\geq 95^{\text {th }}$

C

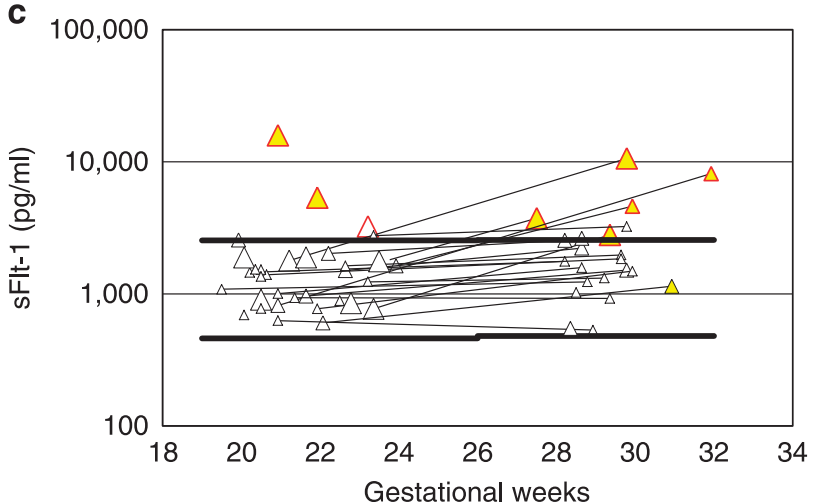

d

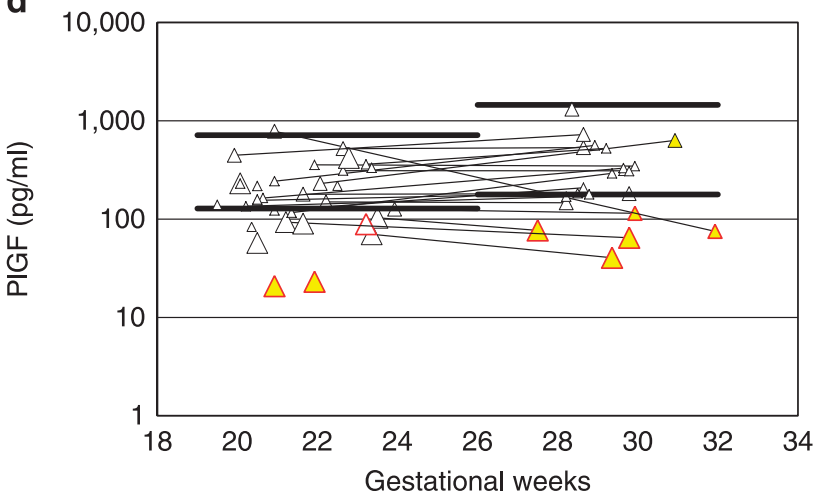

Figure 1 False-positive and true-positive cases for predicting the imminent onset of preeclampsia (PE) using the three-step approach. The meanings of the symbols are presented in the figures. In brief, the symbols accentuated by a bold red line indicate cases that are maternal factors (MF) (either systolic blood pressure $\geqslant 120 \mathrm{~mm} \mathrm{Hg}$ or diastolic blood pressure $\geqslant 80 \mathrm{~mm} \mathrm{Hg}$ or past history of PIH) and with a soluble fms-like tyrosine kinase 1 (sFlt-1) level in the $\geqslant 95$ th percentile and a placental growth factor (PIGF) level in the $<5$ th percentile. The false-positive cases ( $\bigcirc$, normal pregnant women; $\square$, women with gestational hypertension (GH)) are shown in $\mathbf{a}$ and $\mathbf{b}$. The true positive cases (women with imminent onset) are represented by closed yellow triangles $(\mathbf{\Lambda})$, and the false positive cases (women with PE, but not imminent onset) are represented by open triangles accentuated by red bold line ( $\Delta$ ), in $\mathbf{c}$ and $\mathbf{d}$. (a) shows the raw values of sFlt-1 at 19-31 weeks of gestation in 1194 normal pregnant women and 20 women with GH in a prospective cohort study. (b) shows the raw values of PIGF at 19-31 weeks of gestation in 1194 normal pregnant women and 20 women with GH in a prospective cohort study. (c) shows the raw values of sFlt-1 at 19-31 weeks of gestation in 35 preeclamptic women before the onset of PE in a prospective cohort study. (d) shows the raw values of PIGF at 19-31 weeks of gestation in 35 preeclamptic women before the onset of PE in a prospective cohort study. In a-d, the thick straight lines represent the 5th and 95th percentiles of the distribution of the $\log _{10}$ sFIt-1 and $\log _{10}$ PIGF in 1155 normal pregnant women at 19-25 weeks of gestation and 769 normal pregnant women at $26-31$ weeks of gestation in a prospective cohort study. When the plasma level of sFlt- 1 is $\geqslant 95$ th percentile, the sFlt- 1 is abnormal; and when the plasma level of PIGF is $<5$ th percentile, the PIGF is abnormal. In $\mathbf{c}$ and $\mathbf{d}$, the marks in the cases with two samplings at 19-25 and 26-31 weeks of gestation are connected by a thin line. wks, weeks.

target population using the three-step approach using the sFlt-1/PlGF ratio as the onset threshold was reduced by $78 \%$ compared with the approach using the onset threshold of the sFlt-1/PlGF ratio because the subject numbers in the second step were nearly $40 \%$ and subject numbers in the third step were nearly $4 \%$. If we assume the costs of the PlGF and sFlt-1 measurements are 10000 yen each, the average cost for the total population using the three-step approach may be cheaper than that using the onset threshold of the sFlt-1/PlGF ratio (4400 yen vs. 20000 yen), although both approaches achieved high SE, LR + and PPV values. ${ }^{16}$ How should this early diagnostic test using the circulating levels of PlGF and sFlt-1 be used to predict the imminent onset of PE in a clinical practice? We advocate that the circulating levels of PlGF and sFlt-1 should be measured in women with a $\mathrm{BP} \geqslant 120 / 80 \mathrm{~mm} \mathrm{Hg}$ or a past history of $\mathrm{PE} / \mathrm{GH}$, based on the current diagnostic power of the three-step approach with an SE of $>0.80$, an LR + of $>90$ and a PPV of $>0.40$. Actually, our threestep method could not predict the imminent onset of PE that occurred in some women with a BP $<120 / 80 \mathrm{~mm} \mathrm{Hg}$; however, we speculate that the risk of an imminent onset of $\mathrm{PE}$ at $<34$ weeks of gestation in the women with a $\mathrm{BP}<120 / 80 \mathrm{~mm} \mathrm{Hg}$ may be very low because only one women (9\%) with early-onset PE showed a BP $<120 / 80 \mathrm{~mm} \mathrm{Hg}$ at $16-23$ weeks of gestation. 

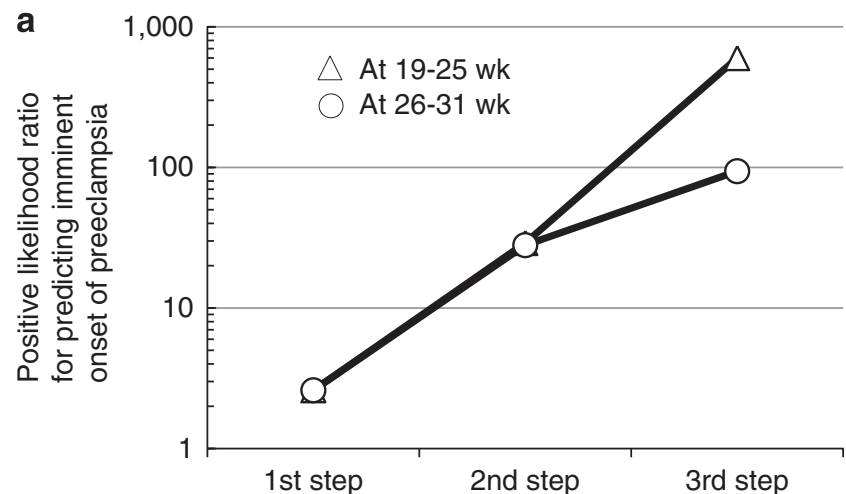

b

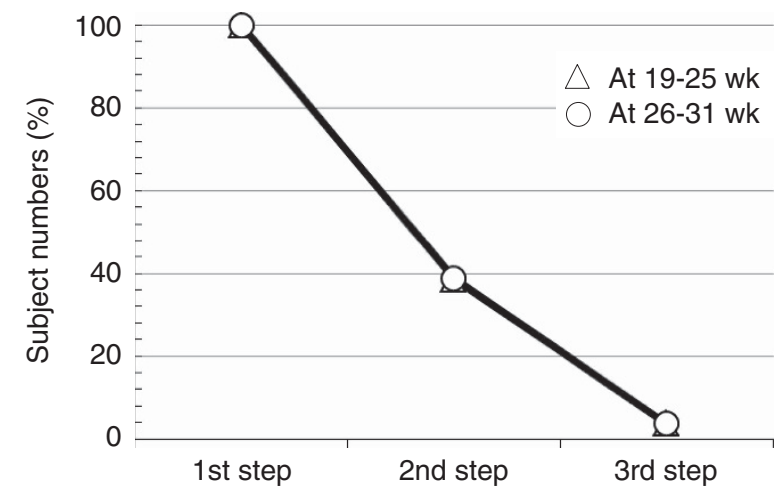

Figure 2 Screening performance using the three-step approach. (a) shows the effects of the three-step approach (maternal factors (first step) $\rightarrow$ PIGF (second step) $\rightarrow$ sFlt-1 (third step)) on the marked changes in the LR + for predicting the imminent onset of $\mathrm{PE}$ at $19-25$ weeks (open triangles) and 26-31 weeks (open circles). (b) shows the effects of the three-step approach on the reduction of the percentages of subject numbers at 19-25 weeks (open triangles) and 26-31 weeks (open circles). At 19-25 weeks, the number of women in each step was $1199,463(39 \%)$ and $44(3.7 \%)$, respectively; and at 26-31 weeks, 798, 310 (39\%) and 29 (3.6\%), respectively.

Our research has a significant limitation. While the SE, LR + and PPV values in the two-step or three-step approach for the imminent onset of PE were good, the number of women with an imminent onset of PE were relatively small, only eight. Because we planned to draw blood samples at 20-23 and 28-29 weeks in our study design, the time between the two samplings was relatively long, almost 8 weeks, resulting in several missed outcomes of PE for predicting the imminent onset of PE within 4 weeks after the blood sampling. We are now planning a new study protocol with blood sampling every 4 weeks from 20 to 35 weeks. In addition, our findings should be confirmed in a larger multicenter, cohort study.

In conclusion, we have developed a three-step approach for predicting the imminent onset of PE with onset at $<4$ weeks after blood sampling during 19-31 weeks of gestation: the first step is a positive selection using either blood pressure levels greater than $120 / 80 \mathrm{~mm} \mathrm{Hg}$ at $20-23$ weeks of gestation or a past history of PIH; the second step is a positive selection using the plasma levels of PlGF at 19-31 weeks of gestation; and the third step is a positive selection using the plasma levels of sFlt-1 at 19-31 weeks of gestation. This approach resulted in an improvement in the cost performance while showing an LR + of $>10$, an SE of $>0.80$ and a PPV of $>0.20$.

\section{CONFLICT OF INTEREST}

The authors declare no conflict of interest.

\section{ACKNOWLEDGEMENTS}

This work was supported by Grants-in-Aid (18591809, 24390383 and 24592482 to AO) from the Ministry of Education, Culture, Sports, Science and Technology in Japan. We greatly thank Roche Diagnostics for kindly providing the Elecsys sFlt-1 and PIGF assays and for their cooperation with the sFlt-1 and PlGF measurements.

1 Shiozaki A, Matsuda Y, Satoh S, Saito S. Impact of fetal sex in pregnancy-induced hypertension and preeclampsia in Japan. J Reprod Immunol 2011; 89: 133-139.

2 Nagaya K, Fetters MD, Ishikawa M, Kubo T, Koyanagi T, Saito Y, Sameshima H, Sugimoto M, Takagi K, Chiba Y, Honda H, Mukubo M, Kawamura M, Satoh S, Neki R. Causes of maternal mortality in Japan. JAMA 2000; 283: 2661-2667.

3 Yamauchi A, Minakami H, Ohkuchi A, Usui R, Idei S, Sato I. Causes of stillbirth: an analysis of 77 cases. J Obstet Gynaecol Res 1999; 25: 419-424.

4 Ohkuchi A, Hirashima C, Matsubara S, Takahashi K, Matsuda Y, Suzuki M. Threshold of soluble fms-like tyrosine kinase 1 /placental growth factor ratio for the imminent onset of preeclampsia. Hypertension 2011; 58: 859-866.

5 Pedrosa AC, Matias A. Screening for preeclampsia: a systematic review of tests combining uterine artery Doppler with other markers. J Perinat Med 2011; 39: 619-635.

6 Espinoza J, Romero R, Nien JK, Gomez R, Kusanovic JP, Gonçalves LF, Medina L, Edwin S, Hassan S, Carstens M, Gonzalez R. Identification of patients at risk for early onset and/or severe preeclampsia with the use of uterine artery Doppler velocimetry and placental growth factor. Am J Obstet Gynecol 2007; 196: e1-13.

7 Stepan H, Unversucht A, Wessel N, Faber R. Predictive value of maternal angiogenic factors in second trimester pregnancies with abnormal uterine perfusion. Hypertension 2007; 49: 818-824.

8 Diab AE, El-Behery MM, Ebrahiem MA, Shehata AE. Angiogenic factors for the prediction of preeclampsia in women with abnormal mid trimester uterine artery Doppler velocimetry. Int J Gynaecol Obstet 2008; 102: 146-151.

9 Poon LC, Kametas NA, Maiz N, Akolekar R, Nicolaides KH. First-trimester prediction of hypertensive disorders in pregnancy. Hypertension 2009; 53: 812-818.

10 Jaeschke R, Guyatt GH, Sackett DL. Uers' guides to the medical literature. III. How to use an article about a diagnostic test. B. What are the results and will they help me in caring for my patients? The Evidence-Based Medicine Working Group. JAMA 1994; 271: 703-707.

11 Conde-Agudelo A, Villar J, Lindheimer M. World Health Organization systematic review of screening tests for preeclampsia. Obstet Gynecol 2004; 104: 1367-1391.

12 Levine RJ, Maynard SE, Qian C, Lim KH, England LJ, Yu KF, Schisterman EF, Thadhani R, Sachs BP, Epstein FH, Sibai BM, Sukhatme VP, Karumanchi SA. Circulating angiogenic factors and the risk of preeclampsia. New Engl J Med 2004; 350: 672-683.

13 Ohkuchi A, Iwasaki R, Suzuki H, Hirashima C, Takahashi K, Usui R, Matsubara S, Minakami H, Suzuki M. Normal and high-normal blood pressures, but not body mass index, are risk factors for the subsequent occurrence of both preeclampsia and gestational hypertension: a retrospective cohort study. Hypertens Res 2006; 29: 161-167.

14 Pewsner D, Battaglia M, Minder C, Marx A, Bucher HC, Egger M. Ruling a diagnosis in or out with "SpPIn" and "SnNOut": a note of caution. BMJ 2004; 329: 209-213.

15 Ohkuchi A, Hirashima C, Suzuki H, Takahashi K, Yoshida M, Matsubara S, Suzuki M. Evaluation of a new and automated electrochemiluminescence immunoassay for plasma sFlt-1 and PIGF levels in women with preeclampsia. Hypertens Res 2010; 33: $422-427$.

16 Ohkuchi A, Hirashima C, Takahashi K, Suzuki H, Matsubara S, Suzuki M. Onset threshold of plasma levels of soluble fms-like tyrosine kinase 1 /placental growth factor ratio for predicting imminent onset of preeclampsia within 4 weeks after blood sampling at 19 to 31 weeks of gestation. Hypertens Res 2013; 36: 1073-1080.

17 Takahashi K, Ohkuchi A, Hirashima C, Matsubara S, Suzuki M. Establishing reference values for mean notch depth index, pulsatility index and resistance index in the uterine artery at 16-23 weeks' gestation. J Obstet Gynaecol Res 2012; 38: 1275-1285.

18 Ohkuchi A, Minakami H, Sato I, Mori H, Nakano T, Tateno M. Predicting the risk of preeclampsia and a small-for-gestational-age infant by quantitative assessment of the diastolic notch in uterine artery flow velocity waveforms in unselected women. Ultrasound Obstet Gynecol 2000; 16: 171-178.

19 Sato K. A proposal for a new definition and classification of "pregnancy induced hypertension (PIH)" (2004). In: Japan Society for the Study of Toxemia of Pregnancy (eds). Historical Perspective of Study of Pregnancy-Induced Hypertension in Japan. Medical View Co, Tokyo, Japan, 2005, pp 54-87.

20 Ogawa Y, Iwamura T, Kuriya N, Kuriya N, Nishida H, Takeuchi H, Yakada M, Itabashi K, Imura S, Isobe K. Birth size standards by gestational age for Japanese neonates. Acta Neonatol Jpn 1998; 34: 624-632.

21 Confidence interval calculator [Computer Program]. Available at http://www.pedro.org. au/english/downloads/confidence-interval-calculator/. (Accessed 30 September 2012.). 